\title{
New opportunities of dendrimers for theranostic approaches to personalized medicine
}

Theranostic nanomedicine paradigm, a combination of specific molecular diagnostic tests and specific targeted therapy, involves two topics: therapeutic product efficacies and diagnosis. The first topic concerns identified patients to be treated, whereas the second is to select patients for personalized medicine. Generally, nanotechnology allows developing theranostic strategy with the same nanoparticle. Theranostics represents a new field of medicine and today it is associated with molecular imaging technologies. In reality, theranostic approach has about a history of 75 years since the use, throughout the world, of radioactive iodine for the well-established diagnosis and treatment of thyroid cancer. It is clear that the emergence of new nanotechnologies has opened new opportunities to bring together diagnosis and tailored therapy approaches. Thus, during the past decade, it has been emerging that theranostic strategy is the best way to deliver "the right drug for the right patient at the right moment" to tackle not only cancer but to address unmet medical needs. Importantly, the development of novel imaging strategies revolutionized the nanotheranostic systems. The main multifunctional theranostic platforms are carbon-based nanomaterials, drug conjugates, aliphatic polymers, micelles, vesicles, core-shell nanoparticles, microbubbles and dendrimers bearing different contrast agents and drugs. In particular dendrimers has emerged as a new class of highly tunable hyperbranched polymers. Magnetic resonance imaging, gamma scintigraphy, computed tomography and optical imaging are the main techniques developed with dendrimers.

From these basic scientific perspectives, SCIENCE CHINA Materials organized this special issue themed on "Diagnostic and Theranostic Platforms Based on Dendrimers and Hyperbranched Polymers" to review focused personalized medicines with theranostic approaches. Six very interesting reviews allow defining the frame of the use of dendrimers in the theranostic domain.
From a global view, Shi et al. (https://doi.org/10.1007/ s40843-018-9271-4) report recent advances on the use of dendrimer-based systems for cancer therapy. Surface modifications of dendrimers and the application of dendrimers as versatile platforms to cancer were concerned. Majoral, Mignani and co-workers (https://doi.org/ $10.1007 / \mathrm{s} 40843-018-9244-5)$ present the recent therapeutic applications of the theranostic principle with dendrimers in oncology, while Peng et al. (https://doi.org/ 10.1007/s40843-018-9323-6) highlight the recent progress, opportunities and challenges in developing dendrimer-based MRI contrast agents for brain tumor imaging. More focused review is from Jiang et al. (https:// doi.org/10.1007/s40843-018-9242-3) concerning the recent studies on dendrimer-based nanoparticles in cancer chemotherapy and gene therapy. This review highlights the studies of the specific controlled release of anti-cancer agents to tumor tissues and cells in a specific tumor microenvironment. Supramolecular dendritic polymers (SDPs) providing a new opportunity for the precision diagnosis and treatment of diseases have been highlighted by Zhu and co-workers (https://doi.org/10.1007/s40843018-9252-y). Majoral et al. (https://doi.org/10.1007/ s40843-018-9253-5) described a specific third-generation of diagnosis enabling the search of a broad range of pathogens with strong sensitivity and specificity. This approach is developed in the French company named Dendris.

Three more specific research papers describe the use of dendrimers or dendritic polymers to tackle cancer. Luo and Gu et al. (https://doi.org/10.1007/s40843-018-9277-8) present the development of enzyme/pH-sensitive dendritic polymer-DOX conjugate for cancer treatment, whereas Chen and co-workers (https://doi.org/10.1007/ s40843-018-9238-6) detailed an original study about the simultaneous development of both tumor photothermal therapy and immunotherapy using macrophages loaded 
with CPG and GNR-PEI to treat cancer. Synthesis of water-soluble dye-cored poly(amidoamine) dendrimers for long-term live cell imaging was described by Yin et al. (https://doi.org/10.1007/s40843-018-9246-6).

All the three guest editors would like to acknowledge the renowned authors in the field to contribute this special issue by submitting their state of art reviews and high quality research papers as well as the reviewers for their vigorous and timely comments to ensure the quality of the publications. We would also sincerely thank the editorial team of SCIENCE CHINA Materials to offer such a wonderful opportunity to publish this nice special issue.

\section{Guest editors}

Jean-Pierre Majoral

Professor, Laboratoire de Chimie de Coordination, Centre National de la Recherche Scientifique, Toulouse, France Email: majoral@lcc-toulouse.fr

Xiangyang Shi

Professor, College of Chemistry, Chemical Engineering, and Biotechnology, Donghua University, Shanghai, China

Email: xshi@dhu.edu.cn

Serge Mignani

Professor, CQM-Centro de Química da Madeira, MMRG, Universidade da Madeira, Campus da Penteada, 9020-105 Funchal, Portugal

Email: Serge.mignani@staff.uma.pt 